\title{
Balancedness conditions for exact games
}

Citation for published version (APA):

Csóka, P., Herings, P. J. J., \& Kóczy, L. Á. (2007). Balancedness conditions for exact games. METEOR, Maastricht University School of Business and Economics. METEOR Research Memorandum No. 040 https://doi.org/10.26481/umamet.2007040

Document status and date:

Published: 01/01/2007

DOI:

10.26481/umamet.2007040

Document Version:

Publisher's PDF, also known as Version of record

\section{Please check the document version of this publication:}

- A submitted manuscript is the version of the article upon submission and before peer-review. There can be important differences between the submitted version and the official published version of record.

People interested in the research are advised to contact the author for the final version of the publication, or visit the DOI to the publisher's website.

- The final author version and the galley proof are versions of the publication after peer review.

- The final published version features the final layout of the paper including the volume, issue and page numbers.

Link to publication

\footnotetext{
General rights rights.

- You may freely distribute the URL identifying the publication in the public portal. please follow below link for the End User Agreement:

www.umlib.nl/taverne-license

Take down policy

If you believe that this document breaches copyright please contact us at:

repository@maastrichtuniversity.nl

providing details and we will investigate your claim.
}

Copyright and moral rights for the publications made accessible in the public portal are retained by the authors and/or other copyright owners and it is a condition of accessing publications that users recognise and abide by the legal requirements associated with these

- Users may download and print one copy of any publication from the public portal for the purpose of private study or research.

- You may not further distribute the material or use it for any profit-making activity or commercial gain

If the publication is distributed under the terms of Article $25 \mathrm{fa}$ of the Dutch Copyright Act, indicated by the "Taverne" license above, 
Péter Csóka, P. Jean-Jacques Herings,

László Á. Kóczy

Balancedness Conditions for Exact Games

$\mathrm{RM} / 07 / 040$

JEL code: C71, C61

\section{METE@R}

Maastricht research school of Economics of TEchnology and ORganizations

Universiteit Maastricht

Faculty of Economics and Business Administration P.O. Box 616

NL - 6200 MD Maastricht

phone : ++31433883830

fax : :+31433884873 


\title{
Balancedness Conditions for Exact Games
}

\author{
Péter Csóka* P. Jean-Jacques Herings ${ }^{\dagger} \quad$ László Á. Kóczy
}

October 5, 2007

\begin{abstract}
We provide two new characterizations of exact games. First, a game is exact if and only if it is exactly balanced; and second, a game is exact if and only if it is totally balanced and overbalanced.

The condition of exact balancedness is identical to the one of balancedness, except that one of the balancing weights may be negative while for overbalancedness one of the balancing weights is required to be non-positive and no weight is put on the grand coalition. Exact balancedness and overbalancedness are both easy to formulate conditions with a natural game-theoretic interpretation and are shown to be useful in applications.

Using exact balancedness we show that exact games are convex for the grand coalition and that the classes of convex and totally exact games coincide. We provide an example of a game that is totally balanced and convex for the grand coalition, but not exact. Finally we relate classes of balanced, totally balanced, convex for the grand coalition, exact, totally exact, and convex games to one another.

Keywords: Totally Balanced Games, Exact Games, Convex Games

JEL Classification: C71, C61
\end{abstract}

*Department of Economics, Maastricht University, P.O. Box 616, 6200 MD, Maastricht, The Netherlands. E-mail:P.Csoka@algec.unimaas.nl.

${ }^{\dagger}$ Department of Economics, Maastricht University, P.O. Box 616, 6200 MD, Maastricht, The Netherlands. E-mail:P.Herings@algec.unimaas.nl. The author would like to thank the Netherlands Organisation for Scientific Research (NWO) for financial support.

${ }^{\ddagger}$ Department of Economics, Maastricht University, P.O. Box 616, 6200 MD, Maastricht, The Netherlands. E-mail:L.Koczy@algec.unimaas.nl. The author would like to thank funding by the European Union under the Marie Curie Intra-European Fellowship MEIFCT-2004-011537. 


\section{Introduction}

It is well-known that the core in a transferable utility game is non-empty if and only if the game is balanced (Bondareva, 1963; Shapley, 1967). The core of every subgame of a transferable utility game is non-empty if and only if the game is totally balanced. Totally balanced games arise from a wide range of applications. They coincide with market games (Shapley and Shubik, 1969); also with a special case of market games with a continuum of indivisible commodities: cooperation in fair division (Legut, 1990); they are equivalent

to a class of maximum flow problems (Kalai and Zemel, 1982a); and also to permutation games of less than four players (Tijs, Parthasarathy, Potters, and Prassad, 1984). Moreover, totally balanced games are generated by linear production games (Owen, 1975), generalized network problems (Kalai and Zemel, 1982b), and controlled mathematical programming problems (Dubey and Shapley, 1984).

In this paper we provide a set of linear programming problems to study a subclass of totally balanced games, exact games (Schmeidler, 1972). By the linear programming problems one can easily check whether a game is exact or not. Using the dual of the linear programming problems we develop two new characterizations of exact games. These characterizations complement earlier characterizations by Schmeidler (1972) and Azrieli and Lehrer (2005).

The first characterization of the class of exact games is the condition of exact balancedness. One interpretation of balancedness is that the players can distribute one unit of working time to any coalition and in doing so cannot generate more value than the grand coalition. The condition of exact balancedness is identical to the one of balancedness, except that one coalition is allowed to exist for a negative amount of time, which would allow its players to spend more than one unit of time in the other coalitions. We show that exact balancedness implies total balancedness.

The second characterization spells out what more than total balancedness is needed to obtain exactness. It says that a game is exact if an only if it is totally balanced and overbalanced. In case of overbalancedness, no weight is put on the grand coalition and one coalition exists for a non-positive amount of time, which requires the players to spend at least one unit of time in the other coalitions. From this characterization it follows immediately that an exact game is totally balanced.

The simplicity of our balancedness conditions is helpful in applications. Csóka, Herings, and Kóczy (2007) use total balancedness and overbalancedness to show that risk allocation games with no aggregate uncertainty are exact. Biswas, Parthasarathy, Potters, and Voorneveld (1999) show that totally exact games are convex (and that convex games are totally exact). 
Using exact balancedness we provide an alternative proof of this result.

We also study games that are convex for the grand coalition, where convexity is only required for coalitions whose union is the set of all players. We show that exact balancedness implies that a game is convex for the grand coalition, which leads to the result that exact games of less than four players are convex. Using the intuition behind exact balancedness, we provide an example of a game which is both totally balanced and convex for the grand coalition, but which is not exact.

The structure of the paper is as follows. We start with the notation and the necessary definitions. In Section 3 we study the balancedness conditions for exact games. In Section 4 we demonstrate the usefulness of our characterizations of exact games. We conclude the paper with a summary of how the classes of balanced, totally balanced, convex for the grand coalition, exact, totally exact, and convex games are related to one another.

\section{Notation and Definitions}

Let $N=\{1, \ldots, n\}$ denote a finite set of players, $\mathcal{N}$ is the collection of non-empty subsets of $N$, and $\mathcal{D}$ is the collection of non-empty subsets of a coalition $D \in \mathcal{N}$. A value function $v: 2^{N} \rightarrow \mathbb{R}$ satisfying that $v(\emptyset)=0$ gives rise to a cooperative game with transferable utility (game, for short) $(N, v)$. Let $\Gamma$ denote the set of games with $n$ players. An allocation is a vector $x \in \mathbb{R}^{n}$, where $x_{i}$ is the payoff of player $i \in N$. For a coalition $C \in \mathcal{N}$, let $x(C)=\sum_{i \in C} x_{i}$. An allocation $x \in \mathbb{R}^{n}$ is called efficient if $x(N)=v(N)$, individually rational if $x_{i} \geq v(\{i\})$ for all $i \in N$, and coalitionally rational if $x(C) \geq v(C)$ for all $C \in \mathcal{N}$. The core is the set of efficient and coalitionally rational allocations.

For each $C \in \mathcal{N}, a(C) \in \mathbb{R}^{n}$ is the membership vector in $C$, where $a_{i}(C)=1$ if $i \in C$ and $a_{i}(C)=0$ otherwise.

Definition 2.1. A balanced vector of weights is $\left(\lambda_{C}\right)_{C \in \mathcal{N}}$ such that $\lambda_{C} \in \mathbb{R}_{+}$ and $\sum_{C \in \mathcal{N}} \lambda_{C} a(C)=a(N)$. A game $(N, v)$ is balanced if $\sum_{C \in \mathcal{N}} \lambda_{C} v(C) \leq$ $v(N)$ for all balanced vectors of weights $\left(\lambda_{C}\right)_{C \in \mathcal{N}}$.

Let $\Gamma_{\mathrm{b}}$ denote the class of balanced games with $n$ players. A well-known interpretation of balancedness is that if the players distribute one unit of working time to any coalition and each coalition is active during $\lambda_{C}$ time units, then the players cannot generate more value than $v(N)$, the value of the grand coalition. Balancedness is a necessary and sufficient condition for the core in a transferable utility game to be non-empty (Bondareva, 1963; Shapley, 1967); Predtetchinski and Herings (2004) provide the corresponding 
necessary and sufficient balancedness conditions for non-transferable utility games.

For a game $(N, v)$ and a coalition $C \in \mathcal{N}$ the subgame $\left(C, v^{C}\right)$ is obtained by restricting $v$ to subsets of $C$.

Definition 2.2. A game $(N, v)$ is totally balanced if for every $D \in \mathcal{N}$ its subgame $\left(D, v^{D}\right)$ is balanced, that is, if for all $D \in \mathcal{N}$ and for all vectors $\left(\lambda_{C}\right)_{C \in \mathcal{D}}$ such that $\lambda_{C} \in \mathbb{R}_{+}$and $\sum_{C \in \mathcal{D}} \lambda_{C} a(C)=a(D)$, we have $\sum_{C \in \mathcal{D}} \lambda_{C} v(C) \leq v(D)$.

In a totally balanced game every subgame has a non-empty core. Let $\Gamma_{\mathrm{tb}}$ denote the class of totally balanced games with $n$ players.

Schmeidler (1972) introduces exact games.

Definition 2.3. A game $(N, v)$ is exact if for each $C \in 2^{N}$ there exists a core allocation $x$ such that $x(C)=v(C)$.

Let $\Gamma_{\mathrm{e}}$ denote the class of exact games with $n$ players.

Definition 2.4. A game $(N, v)$ is convex if for all $S, T \in 2^{N}$ we have $v(S)+$ $v(T) \leq v(S \cup T)+v(S \cap T)$.

Let $\Gamma_{\mathrm{c}}$ denote the class of convex games with $n$ players. Convex games are exact (Schmeidler, 1972). We now introduce the new notion of convexity for the grand coalition.

Definition 2.5. A game $(N, v)$ is convex for the grand coalition if for all $S, T \in 2^{N}$ such that $S \cup T=N$ we have $v(S)+v(T) \leq v(N)+v(S \cap T)$.

Let $\Gamma_{\mathrm{cg}}$ denote the set of games which are convex for the grand coalition. Following Biswas, Parthasarathy, Potters, and Voorneveld (1999), we define totally exact games analogously to totally balanced games.

Definition 2.6. A game $(N, v)$ is totally exact if for every $D \in \mathcal{N}$ its subgame $\left(D, v^{D}\right)$ is exact.

Let $\Gamma_{\text {te }}$ denote the class of totally exact games with $n$ players.

\section{Exact Games and Balancedness}

Consider a game $(N, v) \in \Gamma$. For each coalition $D \in \mathcal{N}$ we develop a linear programming problem related to the game $(N, v)$. The linear program is such that whenever $(N, v)$ is exact any optimal solution $x^{*}$ is a core allocation 
satisfying $x^{*}(D)=v(D)$. We denote the linear program by $\mathrm{P}_{v, D}$ and its dual by $\mathrm{D}_{v, D}$.

$$
\begin{aligned}
& \min a(N) x \\
& \text { s. t. } \\
& \max \sum_{C \in \mathcal{N}} \lambda_{C} v(C) \\
& \text { s. t. }
\end{aligned}
$$

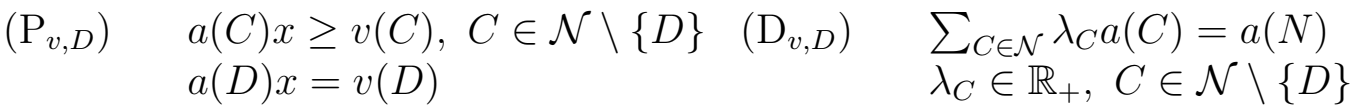

$$
\begin{aligned}
& x \in \mathbb{R}^{n} \\
& \lambda_{D} \in \mathbb{R} \text {. }
\end{aligned}
$$

Let us discuss the primal. The first part of the feasibility constraints requires that $x$ is coalitionally rational for all coalitions but coalition $D$. The second part of the feasibility constraints requires that $x(D)=v(D)$, that is, $x$ should be efficient in the subgame $\left(D, v^{D}\right)$, so a feasible solution yields a core element in the subgame $\left(D, v^{D}\right)$.

If $\left(D, v^{D}\right)$ is a balanced game, then the set of feasible solutions is nonempty, since we have a core element in the subgame $\left(D, v^{D}\right)$ and the other elements of a feasible solution can be chosen sufficiently large. In this case the set of optimal solutions of $\left(\mathrm{P}_{v, D}\right)$ is also non-empty since the set of feasible solutions is bounded from below. If $\left(D, v^{D}\right)$ is not balanced, then the set of feasible solutions of $\left(\mathrm{P}_{v, D}\right)$ is empty, since then $\left(D, v^{D}\right)$ has no core allocations. If $x^{*}$ is an optimal solution of the primal, then $o_{v, D}=a(N) x^{*}$ is the value of the optimal solution, which by the duality theorem of linear programming is the same as the optimal objective value of the dual. If $\left(\mathrm{P}_{v, D}\right)$ has no optimal solutions, then we define $o_{v, D}=\infty$. The linear programming problems are related to exactness as follows.

Proposition 3.1. A game $(N, v) \in \Gamma$ is exact if and only if for every $D \in \mathcal{N}$ we have that $o_{v, D}=v(N)$.

\section{Proof.}

$(\Rightarrow)$ The proof is by contradiction. Take any exact game $(N, v) \in \Gamma_{\mathrm{e}}$ and assume that there is a coalition $D \in \mathcal{N}$ such that $o_{v, D} \neq v(N)$. Let $x^{*}$ be an optimal solution of $\left(\mathrm{P}_{v, D}\right)$. Using the feasibility constraints of the primal we have that $A(N) x^{*}>v(N)$. As $(N, v)$ is exact there exists a core element $x$ such that $x(D)=v(D)$ and $x(N)=v(N)$. The vector $x$ satisfies the feasibility constraints and has objective value $A(N) x=v(N)<A(N) x^{*}$, a contradiction.

$(\Leftarrow)$ Assume that for each $D \in \mathcal{N}$ the value of the optimal solution is $v(N)$. Let $x^{*}$ be an optimal solution of $\left(\mathrm{P}_{v, D}\right)$, so $A(N) x^{*}=v(N)$, that is $x^{*}$ is efficient. The efficiency and the feasibility constraints imply that $x^{*}$ is a core allocation of $(N, v)$ with $x(D)=v(D)$. Thus $(N, v)$ is exact. 
Let us continue by analyzing the dual, $\left(\mathrm{D}_{v, D}\right)$. It follows from optimality that $o_{v, D}=v(N)$ if and only if for all feasible solutions of $\left(\mathrm{D}_{v, D}\right)$ the value of the objective function is not larger than $v(N)$. Using this observation, Proposition 3.1, and the linear programming problems we have the following corollary.

Corollary 3.2. A game $(N, v) \in \Gamma$ is exact if and only if for every $D \in \mathcal{N}$ and for each vector $\left(\lambda_{C}\right)_{C \in \mathcal{N}}$ such that for $C \in \mathcal{N} \backslash\{D\}, \lambda_{C} \in \mathbb{R}_{+}, \lambda_{D} \in \mathbb{R}$, and $\sum_{C \in \mathcal{N}} \lambda_{C} a(C)=a(N)$ we have $\sum_{C \in \mathcal{N}} \lambda_{C} v(C) \leq v(N)$.

In Theorem 3.4 below, we rewrite the conditions in Corollary 3.2 using exact balancedness, defined as follows.

Definition 3.3. An exactly balanced vector of weights is a vector $\left(\lambda_{C}\right)_{C \in \mathcal{N}}$ such that for some $D \in \mathcal{N}, \lambda_{D} \in \mathbb{R}$, for $C \neq D, \lambda_{C} \in \mathbb{R}_{+}$, and $\sum_{C \in \mathcal{N}} \lambda_{C} a(C)=$ $a(N)$. A game $(N, v)$ is exactly balanced if $\sum_{C \in \mathcal{N}} \lambda_{C} v(C) \leq v(N)$ for all exactly balanced vectors of weights.

Notice that the only difference to the condition of balancedness is that one weight, $\lambda_{D}$ can be arbitrary (negative, zero, or positive). Exact balancedness is a necessary and sufficient condition for a game to be exact, as the following theorem claims.

Theorem 3.4. A game $(N, v) \in \Gamma$ is exact if and only if it is exactly balanced.

Proof. This follows immediately from Corollary 3.2.

Exact games can also be characterized by total balancedness and overbalancedness, to be defined next.

Definition 3.5. An overbalanced vector of weights is a vector $\left(\mu_{C}\right)_{C \in 2^{N} \backslash\{\emptyset, N\}}$ such that $\mu_{C} \in \mathbb{R}_{+}$and $\sum_{C \in \mathcal{N} \backslash\{D, N\}} \mu_{C} a(C)=a(N)+\mu_{D} a(D)$ for some $D \in$ $\mathcal{N}$. A game $(N, v)$ is overbalanced if $\sum_{C \in \mathcal{N} \backslash\{D, N\}} \mu_{C} v(C) \leq v(N)+\mu_{D} v(D)$ for all overbalanced vectors of weights.

The difference between balancedness and overbalancedness is that in the case of overbalancedness, no weight is put on the grand coalition and one coalition is forced to exist for a non-positive amount of time $\left(-\mu_{D} \leq 0\right)$, which requires players to spend at least one unit of time in the other coalitions.

Theorem 3.6. A game $(N, v) \in \Gamma$ is exact if and only if it is totally balanced and overbalanced. 
Proof. We show that a game $(N, v) \in \Gamma$ is totally balanced and overbalanced if and only if it is exactly balanced.

$(\Leftarrow)$ For $D \in \mathcal{N} \backslash\{N\}$ balancedness of $\left(D, v^{D}\right)$ is obtained by setting the exactly balanced weights such that $\lambda_{N}=1$ and $\lambda_{D}=-1$. Balancedness of $(N, v)$ is obtained by considering exactly balanced vectors of weights where all the weights are non-negative. Using exactly balanced vectors of weights with $\lambda_{N}=0$ and $\lambda_{D} \leq 0$ implies overbalancedness of $(N, v)$.

$(\Rightarrow)$ Take any exactly balanced vector of weights $\left(\lambda_{C}\right)_{C \in \mathcal{N}}$ with $\lambda_{D} \in \mathbb{R}$, $\lambda_{C} \in \mathbb{R}_{+}$for $C \in \mathcal{N} \backslash\{D\}$, and

$$
\sum_{C \in \mathcal{N}} \lambda_{C} a(C)=a(N) .
$$

It follows immediately that $\lambda_{N} \leq 1$. We discuss two cases depending on the value of $\lambda_{N}$.

1. $\lambda_{N}<1$. In this case (1) can be rearranged as

$$
\sum_{C \in \mathcal{N} \backslash\{N\}} \frac{\lambda_{C}}{1-\lambda_{N}} a(C)=a(N) .
$$

If $\lambda_{D} \leq 0$, then exact balancedness is implied by overbalancedness of $(N, v)$, otherwise by balancedness of $(N, v)$.

2. $\lambda_{N}=1$. If $\lambda_{D} \geq 0$, then exact balancedness is trivially satisfied since (1) implies $\left(\lambda_{C}\right)_{C \in \mathcal{N} \backslash\{N\}}=0$. If $\lambda_{D}<0$, then (1) can be rearranged as

$$
\sum_{C \in \mathcal{N} \backslash\{D, N\}} \frac{\lambda_{C}}{-\lambda_{D}} a(C)=a(D),
$$

and exact balancedness follows from total balancedness of $(N, v)$.

Theorem 3.6 characterizes exact games as being the only totally balanced and overbalanced games. It follows immediately that an exact game is totally balanced, which was also shown by Schmeidler (1972). The result shows that exact games constitute the subset of totally balanced games that satisfies the extra condition of overbalancedness, where one weight should be non-positive when checking the "normal" balancedness of the game.

Schmeidler (1972) characterizes exact games as follows (see also Derks and Reijnierse (1998), Theorem 7). 
Theorem 3.7. A game $(N, v) \in \Gamma$ is exact if and only if for every $D \in \mathcal{N} \backslash$ $\{N\}$, for each vector $\left(\gamma_{C}\right)_{C \in \mathcal{N}}$ such that $\gamma_{C} \in \mathbb{R}_{+}$and $\sum_{C \in \mathcal{N} \backslash\{N\}} \gamma_{C} a(C)=$ $a(D)+\gamma_{N} a(N)$ we have $\sum_{C \in \mathcal{N} \backslash\{N\}} \gamma_{C} v(C) \leq v(D)+\gamma_{N} v(N)$.

We provide a direct proof of the equivalence of Schmeidler's characterization of exactness and the characterization in Theorem 3.6.

Proposition 3.8. A game $(N, v) \in \Gamma$ satisfies the conditions in Theorem 3.7 if and only if it is totally balanced and overbalanced.

\section{Proof.}

$(\Rightarrow)$ That $\left(D, v^{D}\right)$ is balanced for $D \in \mathcal{N} \backslash\{N\}$ follows by setting $\gamma_{N}=0$. Balancedness of $(N, v)$ follows from weights such that $\gamma_{N}=1$ and $\gamma_{D} \geq 1$. Overbalanced weights satisfy the equality

$$
\sum_{C \in \mathcal{N} \backslash\{D, N\}} \mu_{C} a(C)=a(N)+\mu_{D} a(D) .
$$

The case where $\mu_{D}=0$ follows from Schmeidler's characterization with $\gamma_{N}=$ 1 and $\gamma_{D}=1$. In case $\mu_{D}>0$, (4) is equivalent to

$$
\sum_{C \in \mathcal{N} \backslash\{D, N\}} \frac{\mu_{C}}{\mu_{D}} a(C)=\frac{1}{\mu_{D}} a(N)+a(D) .
$$

It is now easily seen that this case is implied by Schmeidler's characterization when we choose $\gamma_{D}=0, \gamma_{N}=1 / \mu_{D}$, and $\gamma_{C}=\mu_{C} / \mu_{D}$ for $C \in \mathcal{N} \backslash\{D, N\}$.

$(\Leftarrow)$ The balancing weights of Schmeidler satisfy

$$
\sum_{C \in \mathcal{N} \backslash\{N\}} \gamma_{C} a(C)=a(D)+\gamma_{N} a(N) .
$$

In this case Schmeidler's condition is $\sum_{C \in \mathcal{N} \backslash\{N\}} \gamma_{C} v(C) \leq v(D)+\gamma_{N} v(N)$. If $\gamma_{N}=0$, then balancedness of $\left(D, v^{D}\right)$ gives rise to Schmeidler's condition. If $\gamma_{N}>0$ and $\gamma_{D} \geq 1$, then (5) is equivalent to

$$
\sum_{C \in \mathcal{N} \backslash\{D, N\}} \frac{\gamma_{C}}{\gamma_{N}} a(C)+\frac{\gamma_{D}-1}{\gamma_{N}} a(D)=a(N),
$$

and Schmeidler's condition follows from balancedness. If $\gamma_{N}>0$ and $\gamma_{D}<1$, then (5) is equivalent to

$$
\sum_{C \in \mathcal{N} \backslash\{D, N\}} \frac{\gamma_{C}}{\gamma_{N}} a(C)=a(N)+\frac{1-\gamma_{D}}{\gamma_{N}} a(D),
$$


so Schmeidler's condition follows from overbalancedness.

For a coalition $C \in \mathcal{N}$ let $|C|$ denote the number of players involved in the coalition. Azrieli and Lehrer (2005) give the following necessary and sufficient conditions for exactness.

Theorem 3.9. (Azrieli and Lehrer, 2005, Proposition 2) A game $(N, v) \in \Gamma$ is exact if and only if for every $D \in \mathcal{N}$, for each vector $\left(\alpha_{C}\right)_{C \in \mathcal{N}}$ such that $\alpha_{C} \in \mathbb{R}_{+}, \sum_{C \in \mathcal{N}} \alpha_{C}=1, \beta \in[0,1]$ and $\sum_{C \in \mathcal{N}} \alpha_{C} \frac{a(C)}{|C|}=\beta \frac{a(D)}{|D|}+(1-\beta) \frac{a(N)}{|N|}$ we have $\sum_{C \in \mathcal{N}} \alpha_{C} \frac{v(C)}{|C|} \leq \beta \frac{v(D)}{|D|}+(1-\beta) \frac{v(N)}{|N|}$.

Notice that in Theorem 3.9 if $D=N$, we have the usual balancedness condition expressed in terms of average worth, that is when distributing one unit of working time to any coalition, players cannot generate more value per person than in the grand coalition.

In a similar way as in the proof of Proposition 3.8, one can show that the characterization by Azrieli and Lehrer (2005) is equivalent to the characterization by total balancedness and overbalancedness.

Of all the characterizations presented, exact balancedness is closest to balancedness. In fact, the only difference is that in exact balancedness one of the balancing weights can be chosen to be negative. The characterization of exact games as the ones that satisfy total balancedness and overbalancedness spells out what more than total balancedness is needed to obtain exactness.

Our characterizations of exact games differ from the ones by Schmeidler (1972) and by Azrieli and Lehrer (2005) in that a weight of one is put on $a(N)$. This way we can employ the usual interpretation of balancedness, where players allocate one unit of time over the various coalitions, and in doing so cannot generate more value than the grand coalition.

\section{Applications of Exact Balancedness}

Csóka, Herings, and Kóczy (2007) use the characterization of exact games as those that are totally balanced and overbalanced to show that risk allocation games with no aggregate uncertainty are exact. In this section we show a number of applications of the condition of exact balancedness (Definition 3.3). First we demonstrate that exact games are convex for the grand coalition (Definition 2.5) and the class of totally exact games (Definition 2.6) coincides with the class of convex games (Definition 2.4). Then we discuss games with less than four players, where exact games turn out to be convex. Finally, we present an example of a game with four players that is totally balanced, convex for the grand coalition, but not exact. 
Proposition 4.1. If the game $(N, v)$ is exact, then it is convex for the grand coalition, $\Gamma_{\mathrm{e}} \subseteq \Gamma_{\mathrm{cg}}$.

Proof. Take two coalitions $S, T \in 2^{N}$ such that $S \cup T=N$. Let $D=S \cap T$. Since $(N, v)$ is exact, by Theorem 3.4 it is exactly balanced. We define and exactly balanced vector of weights by setting $\lambda_{S}=1, \lambda_{T}=1$, and $\lambda_{D}=-1$. All other balancing weights are set to zero. Notice that this constitutes and exactly balanced vector of weights, since $a(S)+a(T)-a(D)=a(N)$. By exact balancedness we have that $v(S)+v(T)-v(D) \leq v(N)$, that is $v(S)+v(T) \leq v(N)+v(S \cap T)$.

Theorem 4.2. A game is totally exact if and only if it is convex, $\Gamma_{\mathrm{te}}=\Gamma_{\mathrm{c}}$.

\section{Proof.}

$(\Rightarrow)$ Since by Proposition 4.1 exact games are convex for the grand coalition, totally exact games are convex for all coalitions, that is they are convex.

$(\Leftarrow)$ It is known that convex games are exact (Schmeidler, 1972). Since a subgame of a convex game is also convex, we have total exactness.

The following result claims that a game with less than four players that is totally balanced and convex for the grand coalition has to be convex.

Proposition 4.3. Assume that there are less than four players. If the game $(N, v)$ is totally balanced and convex for the grand coalition, then it is convex.

Proof. For one or two player games the claim requires no proof. Consider the case with three players. For coalitions $S, T \in 2^{N}$ such that $S \cup T=N$ convexity follows from the convexity for the grand coalition. When $S \cap T=\{\emptyset\}$, $S \cup T=C$, where $C$ is a two-player coalition, convexity follows from total balancedness. When $S \subseteq T$, where $T$ is a two-player coalition, convexity follows since $S \cap T=S$ and $S \cup T=T$.

Exact games are totally balanced (Theorem 3.6) and convex for the grand coalition (Proposition 4.1): $\Gamma_{\mathrm{e}} \subseteq \Gamma_{\mathrm{tb}} \cap \Gamma_{\mathrm{cg}}$. Since a convex game is exact, using Proposition 4.3 we have the following corollary.

Corollary 4.4. Assume that there are less than four players. Then a game is exact if and only if it is convex.

There exist games that are convex for the grand coalition, but that are not totally balanced, and games that are totally balanced, but not convex for the grand coalition, so $\Gamma_{\text {tb }} \cap \Gamma_{\text {cg }} \subsetneq \Gamma_{\text {tb }}$ and $\Gamma_{\text {tb }} \cap \Gamma_{\text {cg }} \subsetneq \Gamma_{\text {cg }}$. Theorem 3.6 
and Proposition 4.1 imply that $\Gamma_{\mathrm{e}} \subseteq \Gamma_{\mathrm{tb}} \cap \Gamma_{\mathrm{cg}}$. We consider next the question whether there are games that are totally balanced and convex for the grand coalition, but not exact. Using the intuition behind exact balancedness we provide an example of such a game, thus we have that $\Gamma_{\mathrm{e}} \subsetneq \Gamma_{\mathrm{tb}} \cap \Gamma_{\mathrm{cg}}$.

Example 4.5. We present a game that is totally balanced and convex for the grand coalition, but not exact. By Proposition 4.3 we need at least four players. Consider the following game with four players. Let

$$
\begin{aligned}
& v(\{1\})=v(\{2\})=v(\{3\})=v(\{4\})=0, \\
& v(\{1,2\})=v(\{1,3\})=v(\{1,4\})=1, \\
& v(\{2,3\})=v(\{2,4\})=v(\{3,4\})=0, \\
& v(\{1,2,3\})=v(\{1,2,4\})=v(\{1,3,4\})=1, \\
& v(\{2,3,4\})=0, \\
& v(\{1,2,3,4\})=2 .
\end{aligned}
$$

The game $(N, v)$ is totally balanced, since every subgame $\left(C, v^{C}\right)$ where $C$ contains player 1 has a core element where player 1 receives $v(C)$ and the other players nothing, and the zero vector is a core element of all other subgames. The game $(N, v)$ is convex for the grand coalition as $v(N)=2$ weakly exceeds the sum of the value of any other two coalitions. We show that the game $(N, v)$ is not exactly balanced. Let $D=(\{1\})$ and consider the weights $\lambda_{\{1,2\}}=\lambda_{\{1,3\}}=\lambda_{\{1,4\}}=1, \lambda_{\{1\}}=-2$, and set all other weights equal to zero. These weights constitute and exactly balanced vector of weights, since $a(\{1,2\})+a(\{1,3\})+a(\{1,4\})-2 a(\{1\})=a(\{1,2,3,4\})$. However, $v(\{1,2\})+v(\{1,3\})+v(\{1,4\})-2 v(\{1\})=3>2=v(\{1,2,3,4\})$, thus $v$ is not exact.

\section{Conclusion}

In this paper we have provided two new characterizations of exact games using linear programming formulations. The first characterization of exactness is by exact balancedness. The condition of exact balancedness is identical to the one of balancedness, except that exactly one of the weights is allowed to be negative. Using the usual interpretation of balancedness, this is equivalent to saying that players are allowed to spend a negative amount of time in a particular coalition, which enables them to spend more than one unit of time in the other coalitions.

The second one says that a game is exact if an only if it is totally balanced and overbalanced. The condition of overbalancedness requires one coalition 
to exist for a non-positive amount of time, which requires players to spend at least one unit of time in the other coalitions.

By applying exact balancedness we have shown that the class of totally exact games $\left(\Gamma_{\text {te }}\right)$ coincides with the class of convex games $\left(\Gamma_{\mathrm{c}}\right)$. We have also proven that with less than four players the class of exact games coincides with the class of convex games.

Our characterization of exact games by total balancedness and overbalancedness implies that an exact game is totally balanced $\left(\Gamma_{\mathrm{tb}}\right)$, and we have shown that exact balancedness implies that an exact game is convex for the grand coalition $\left(\Gamma_{\mathrm{cg}}\right)$. Finally, we have provided an example of a game that is totally balanced and convex for the grand coalition, but not exact. The relationships between the various classes of games are summarized in Figure 1.

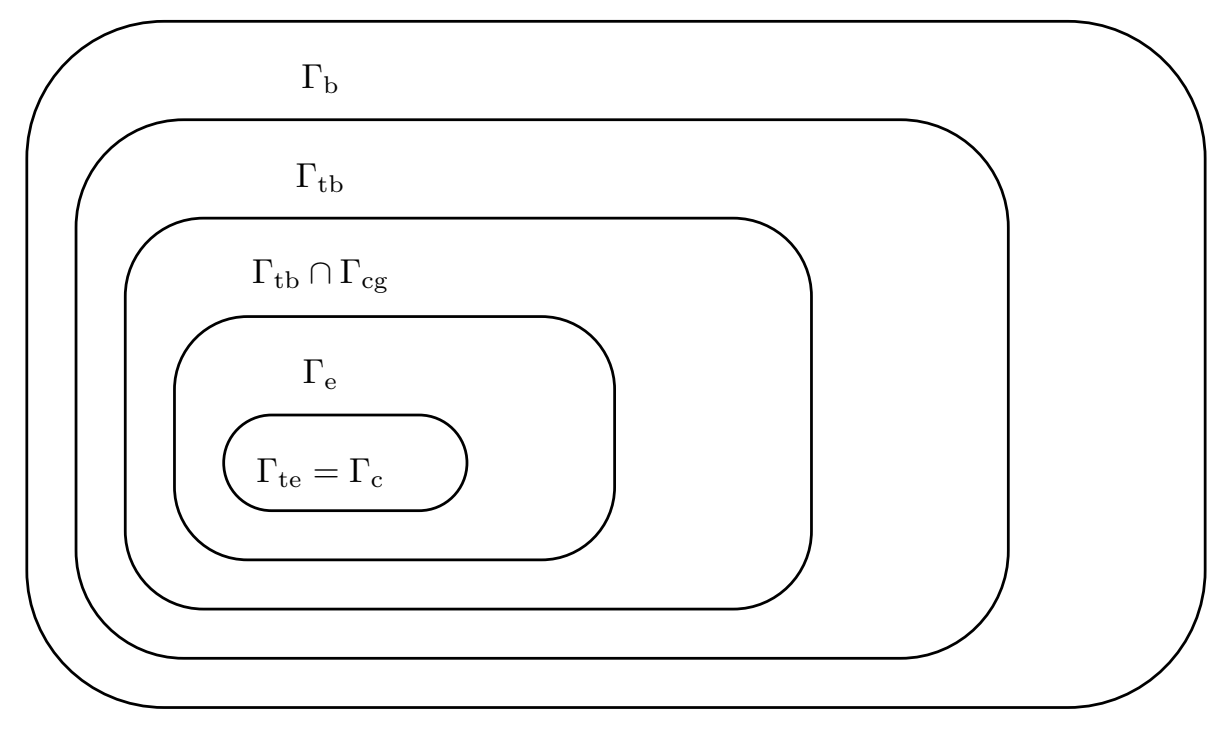

Figure 1: Subsets of balanced games.

\section{References}

Azrieli, Y., Lehrer, E., 2005. Concavification and convex games. Working Paper.

Biswas, A. K., Parthasarathy, T., Potters, J. A. M., Voorneveld, M., 1999. Large cores and exactness. Games and Economic Behavior 28, 1-12. 
Bondareva, Olga N., 1963. Some applications of linear programming methods to the theory of cooperative games (in Russian). Problemy Kybernetiki 10, 119-139.

Csóka, P., Herings, P. J. J., Kóczy, L. Á., 2007. Stable allocations of risk. METEOR Research Memorandum RM07/041, 1-19.

Derks, J., Reijnierse, H., 1998. On the core of a collection of coalitions. International Journal of Game Theory 27, 451-459.

Dubey, P., Shapley, L. S., 1984. Totally balanced games arising from controlled programming problems. Mathematical Programming 29, 245-267.

Kalai, E., Zemel, E., 1982a. Generalized network problems yielding totally balanced games. Operations Research 30, 998-1008.

Kalai, E., Zemel, E., 1982b. Totally balanced games and games of flow. Mathematics of Operations Research 7, 476-478.

Legut, J., 1990. On totally balanced games arising from cooperation in fair division. Games and Economic Behavior 2, 47-60.

Owen, G., 1975. On the core of linear production games. Mathematical Programming $9,358-370$.

Predtetchinski, A., Herings, P. J. J., 2004. A necessary and sufficient condition for the non-emptiness of the core of a non-transferable utility game. Journal of Economic Theory 116, 84-92.

Schmeidler, D., 1972. Cores of exact games. Journal of Mathematical Analysis and Applications 40, 214-225.

Shapley, L. S., 1967. On balanced sets and cores. Naval Research Logistics Quarterly 14, 453-460.

Shapley, L. S., Shubik, M., 1969. On market games. Journal of Economic Theory 1, 9-25.

Tijs, S., Parthasarathy, T., Potters, J., Prassad, V. Rajendra, 1984. Permutation games: Another class of totally balanced games. OR Spektrum 6, 119-123. 